\title{
INCOGNITUS SCRIPTOR: GISANDRA Y GENÉTICA DE LA NATURALEZA EN CARLOS DE OLIVEIRA ${ }^{1}$
}

Pedro SERRA

Universidad de Salamanca

La gisandra, figura central de la novela Finisterra. Paisaje y poblamiento (1978) de Carlos de Oliveira, es un objeto intratable, teológico, verdadero desafío hermenéutico, acaso incluso un desafío a la hermenéutica. Luís Mourão, justamente, ha leído la gisandra como «principio anti-dialéctico»: «forma de pensar el impensable marxista», al significar «la negatividad teleológica de la materialidad histórica y de la materialidad natural. En el mismo ámago de la dialéctica de la naturaleza», así, «Carlos de Oliveira introduce un principio anti-dialéctico [la gisandra es naturaleza que no produce más naturaleza] que lentamente devora para homogeneizar y paralizar» (1996: 285). En la propuesta de lectura de la gisandra que sigue la entiendo como cifra alegórica tanto de una escritura en trance como de un mundo en trance. El mundo posible que podemos reconocer en Finisterra - una casa, una familia (madre, padre, niño), una amenaza exterior al espacio doméstico figurada en la metamórfica y metastática «gisandra»— es la decantación textual del universo social y del espacio físico (el paisanaje y el paisaje) de la Gândara, que tuvo también su refracción en Casa na Duna, Alcateia, Pequenos Burgueses y Uma Abelha na Chuva. El territorio «gandarês», tierra inmóvil, enclave de pobreza, esterilidad y miseria, es una provincia poblada pero inhóspita. La «naturaleza» se perfila, ahí, como un material que resiste a una ocupación humana que significara cobijo estable, habitación segura.

En la peculiar farmacología de la gisandra es necesario, creo, avanzar con un ejercicio de close reading que diferencie dos o tres gisandras. La novela modula la figura a través de distintas opciones de comparación negativa. Por un lado, la gisandra se diferencia de la mandrágora: «Se frota la gisandra entre los senos; ojalá fuera zumo de mandrágora: alcaloides, excitantes, infatigable farmacopea de fertilidad»(Oliveira, 1978: 117). Esta frase nos dice tan sólo que entre la gisandra y la mandrágora se establece una relación particular. ¿Que significa «ojalá fuera»? ¿Deseo de que la gisandra sea o venga a ser en un futuro como la mandrágora? El enunciado no dice «ojalá sea», más bien «ojalá fuera»: ¿deseo, entonces, de que la gisandra viniese a ser como la mandrágora en el sentido de compartir una unidad perdida primordial, no obstante aún recuperable en el presente? Por otro lado, el «ojalá fuera» puede devolvernos un sentido deceptivo. En la base de la frase, entonces, tendríamos no un juicio

\footnotetext{
${ }^{1}$ Este artículo se publicó previamente en http://www.ilicia.es/glosas1.asp?cod=18.
} 
sintético que afirmaría la similitud entre gisandra y mandrágora; mas bien un juicio analítico que las distingue (De Man, 1996: 174). La gisandra no es la mandrágora, y vice-versa. Ahora bien, la propiedad que las distinguiría sería, precisamente, la de la fertilidad. Dónde la mandrágora es zumo fértil, la gisandra puede o no ser fértil, lo que lanza sobre ella el peso de la probabilidad de ser sustancia estéril.

Hasta aquí un conjunto de especificaciones a partir de la frase citada, que no es, sin embargo, el único lugar donde el texto intenta acercarse a la «naturaleza» de la gisandra. Si en este lugar tenemos el par gemelar gisandra/mandrágora, en otro fragmento de la novela deparamos con un otro gemelo de la gisandra, el óvulo: «Óvulo y gisandra siguen procesos degenerativos propios, corrompen a su modo ciertas leyes (y cierta intimidad) de la genética, pero tienden a instaurar una única tara en los dos reinos más vulnerables» (Oliveira, 1978: 119). Anótese que en los primeros dos acordes de la frase, podemos leer la relación analógica entre óvulo y gisandra: comparten el atributo degenerativo, pero en procesos discretos (es decir, «propios»). Incluso «ciertas leyes de la genética», afectadas por los procesos degenerativos, no tienen por qué ser las mismas leyes genéticas. Por último, el atributo que las indistingue, aquella «única tara». Es en este tercer tiempo que se calcula la identidad de ambos, óvulo y gisandra. El último acorde, por fin, recupera la tensión analógica, los «dos reinos más vulnerables» son nuevamente «propios»: el óvulo contamina el reino animal, la gisandra el reino vegetal.

Esta misma dialéctica —una dialéctica en suspensión, podríamos decir- es la que tenemos en la gemelaridad gisandra/feto. He aquí el lugar de Finisterra en que ambos son asimilados: «Todo aceptable si varios fenómenos no escapasen a la regla, si no indicasen incluso que los objetivos parecen alterarse (sean los que sean). ¿Ejemplos? La gisandra, designada para no diferenciar las especies del segundo reino; el feto, cortando (en el primero) un hilo vital, no diferenciando (por escasez o retroceso) caracteres bastante definidos» (181-182). Anótese, desde ya, que la gisandra contamina explícitamente el «segundo reino» (es decir, el reino vegetal), al mismo tiempo que el feto afecta al «primer reino». En este lugar del texto, por otro lado, el elemento importante es el del desarrollo de la supra citada «única tara», el atributo en que convergen gisandra/óvulo, ahora descrito como proceso de «indiferenciación», que es entonces el predicado que in-distingue gisandra/feto. Por último, señálese que la tensión analógica se mantiene: ambos, feto y gisandra «indiferencian», pero mientras que la gisandra lo hace rasurando especies, el feto agencia un sfumato de los «caracteres bastante definidos».

De momento, tres observaciones: la ontología de la gisandra tiene contornos calcados del reino vegetal; tanto óvulo como feto son como la gisandra en la medida en que, operando dentro de los límites de sus reinos respectivos, son agentes de «indiferenciación»; por último, «indiferenciación» no significa lo mismo en el caso de la gisandra, por un lado, y en el caso del par óvulo/feto, por otro (es decir: una cosa es indiferenciar especies, otra indiferenciar «caracteres bastante definidos»). Sea como fuere, lo que estoy intentando argumentar es que la gisandra, dentro de lo que podemos llamar «paradigma gemelar», se define en un marco relacional. Por otro lado, habrá que hacer una distinción entre la gisandra que entra en ese marco relacional - en ese marco analógico- de la gisandra intratable y no-dialéctica. Para tanto, hay que recuperar el capítulo VII y los dos párrafos finales del 
último capítulo de la novela. Curiosamente, el inicio del capítulo VII y los últimos párrafos del capítulo final coinciden en el nombramiento de esa otra gisandra. Léase, entonces: «El estiércol de esta gelatina ávida, que se alimenta de sí misma, de los residuos posibles, de la humedad que escapa a las cuevas por las raíces de alambre, es la gisandra muerta» (61). El capítulo que describe la gisandra empieza, como vemos, por nombrar la «gisandra muerta». Empieza por el fin de la gisandra, y es de ese después del fin de la gisandra que se dice «estiércol» $\mathrm{y}$ «gelatina». Lo hace como un ser vivo, siendo un cuerpo muerto: antropomorfizada, esta «gisandra muerta» «se alimenta», «escapa» o revela «avidez».

Volveré a la descripción con que continúa el capítulo VII, ahora ya de la «gisandra viva». Pero antes, salto hacia el párrafo final del capítulo, donde regresa la actividad de la «gisandra muerta». La presentación de la gisandra es llevada a cabo en el contexto de las «amenazas del exterior» que hacen peligrar la casa. La gisandra, de hecho, no es la única amenaza, también lo es la «niebla» que es descrita en el inicio de este último párrafo. Así, la gisandra se perfila como un «segundo peligro»: «Pronto se juntará con la niebla un segundo peligro, también obsesivo: el barro de las gisandras, golpeando los cimientos todo el invierno» (63). «Estiércol», «gelatina»y ahora «barro» nombran a la «gisandra muerta». Y, una vez más, el nexo relacional que asimila/distingue gisandra y niebla: ambos «obsesivos». Si recuperamos, por último, los dos párrafos finales de la novela, veremos que es también el trabajo de la «gisandra muerta» el que encierra la obra. Una vez más, la introducción de la gisandra es geminada por la niebla que la antecede: «He exagerado, ciertamente, la importancia del paseo nocturno por la casa y el poder del halo contra las amenazas de afuera. No es sólo la niebla: el barro de las gisandras comienza también a entrar» (193). Sólo en este párrafo final llega la gisandra, sin embargo la Casa ya ha sido afectada tanto por la infiltración de la niebla — que es la amenaza número uno- como por el proceso metamórfico invisible de los materiales de que está hecha. La exposición a la niebla y este proceso «interno» son durativos. De la resistencia de los materiales, por cierto, se dirá incluso que su descomposición, prolongada en el tiempo, habrá eventualmente sido fatal: «Han cambiado imperceptiblemente: décadas y décadas de oscuridad. Metamorfosis invisibles (tal vez las más importantes)» (193; yo subrayo). Si la niebla (que tocó la casa mucho antes de haberlo hecho la gisandra) y las metamorfosis invisibles determinaron la descomposición de la Casa, ¿qué función cumple en el domicídio — si se me permite el neologismo - la «gisandra muerta» que, por cierto, tan sólo empezó a entrar?

Todo se juega, en fin, en el último párrafo de la novela. El cotexto, ahí, es fundamental para entender lo que ocurre en ese lugar terminal de la obra: el párrafo anterior hizo diferentes cosas: relativizó el trabajo negativo de la gisandra por la agencia, también corruptora, de la niebla; relativizó el trabajo negativo de la gisandra por el principio «encriptado» de metamorfosis de los materiales; estableció un marco temporal durativo «largo» para el proceso fatídico de la casa, relativizando así la afección súbita por parte de la recién-llegada gisandra. Ahora bien, ¿que nos dice entonces el último párrafo? Lo transcribo íntegro: «Veo (en un breve fulgor del halo) el gelatinoso borbotón rondando la silla donde estuvo el inspector fiscal (y, a continuación, la mujer de las dos caras). Nace en el vano de la ventana (abajo, junto al rodapié: línea abierta entre dos tablas podridas), se extiende por la alfombra. 
Disuelve (primero) la ceniza que cayó del chal de lana; y después (a la segunda embestida) arrastra los granos de saliva del inspector, que tintinean (y brillan) hasta desaparecer» (194). El último párrafo es como una visión: «Veo». Y el vidente es el personaje del adulto que, sentado a la mesa de viñático, revisa los papeles de la familia. El contenido de la visio, mientras tanto, es la disolución de la ceniza y el arrastre y desaparición de los granos de saliva del inspector fiscal. Y es una visión porque hay una alteración perceptiva, que se objetiva si comparamos la visibilidad de este proceso agenciado por el «borbotón gelatinoso» — «gelatina», recordemos, era uno de los nombres de la «gisandra muerta»— con la invisibilidad de las metamorfosis de los materiales de la casa. El personaje del adulto puede ver la disolución, lo que significa también que puede ver un proceso en régimen acelerado. Como si se tratara de una película en fast motion, en una velocidad tal que concentrara en aquel «breve fulgor del halo» —el instante del trance visual, como se lee en el primer paréntesis del párrafo- todo un proceso agenciado por la «gisandra muerta»: «rondar» la silla, «extenderse» por la alfombra, «disolver» la ceniza y, por fin, «arrastrar» los granos de saliva. En suma, la fulguración de esta visio, de esta alucinación del fast motion es llevada hasta las «últimas palabras»: los granos de saliva «tintinean» (trance auditivo) y «brillan» (trance visual). «Hasta desaparecer»: ¿fagocitación por el «borbotón gelatinoso»? ¿O fade out (es decir: fin del «breve fulgor»)?

Regreso, entonces, al ya mencionado capítulo VII para colocar la siguiente cuestión: ¿funciona del mismo modo la «gisandra viva»? ¿En qué se asemeja y en qué se distingue de la «gisandra muerta»? Veamos, entonces, como es el metabolismo de la «gisandra viva», también objeto de descripción en el capítulo VII. La «gisandra viva», perteneciendo al reino vegetal, es retratada como un aparente dispositivo de reprodución que siguiese un modelo «animal». Efectivamente, si nos detenemos en los términos que integran la descriptio de los atributos de la planta veremos que son colocados al lado de predicados del reino animal. La actividad de la gisandra es crepuscular, depende del «viento», del «aire» o del «frémito» que «Eriza las plantas túmidas de leche; comprime los tallos algodonosos; exprime la savia para las campánulas (carne de hongo). El tejido esponjoso se hincha y los poros, dilatados bajo la presión, rezuman un líquido poco espeso (gotas de sudor despuntando en la piel): tensa y húmeda, la cabeza de la gisandra brilla» (62). Por un lado, las unidades léxicas «plantas», «tallos», «savia»y, por otro, las palabras «carne», «sudor», «cabeza», nos devuelven una planta como un animal. El proceso de la actividad crepuscular — «comprimir», «exprimir», «hinchar», «dilatar», «rezumar»— culmina en la etapa nocturna. Durante la noche la actividad de la planta puede depender o no depender del viento, pues interviene un «principio interior»: «Durante la noche, incluso sin viento, el metabolismo de la gisandra (se ha desencadenado el instinto de eyaculación) decide el resto: la planta gotea hasta la madrugada; después, bajo el roce de la luz, explota en un chorro que la vacía y hace que cuelgue hacia el suelo, exhausta» (62). El ciclo crepúsculo/noche tiene como resultado la explosión eyaculatoria.

Ahora bien, ¿es este vaciado eyaculatorio el que reproduce la planta y agencia su crecimiento? El texto no nos lo autoriza a formular de modo categórico. Nos dice que el ciclo metabólico de la planta depende no sólo del «viento», de los trances crepuscular y nocturno, pero también del ritmo de 
las estaciones del año. Un otro ciclo que se define por la secuencia ternaria «estío» / «otoño» / «invierno». Veamos, entonces. El proceso que culmina en la explosión eyaculatoria ocurre durante el «otoño» según el texto: «Durante el otoño, atravesando el jardín, se oye el suelo estallar (tal la pinocha pisada). Expelido su licor, armazones huecas se llenan de ampollas, revientan acompasadamente» (62). La explosión eyaculatoria es aquí modulada por el sintagma «Expelido su licor». Después del chorro, el vaciado, el agotamiento: «armazones huecas». El texto, no obstante, añade nueva información sobre la actividad de las plantas después del ápice eyaculatorio: «se llenan de ampollas»y, en fin, «revientan acompasadamente». Explosión acompasada: ¿las plantas mueren? No lo sé exactamente, pero la fase que sigue, la que corresponde al «invierno», es aquella que tenemos descrita al inicio del capítulo y que se refiere a la «gisandra muerta». Referencia que utiliza no ya el plural «gisandras» y sí el singular «gisandra». En el «invierno» hay actividad en ese ente nombrado «gisandra muerta», pero se trata de una actividad cuyos atributos o predicados son otros con relación a los atributos y predicados de las «gisandras» otoñales y estivales: «El invierno las amasa, les da elasticidad: y entonces la goma burbujeante se expande contra los muros, las paredes de la casa, digiere insectos, arena, hojas, se insinúa en la gestación general y asimila, a su vez, gérmenes ajenos» (63). Durante el «invierno», la «gisandra muerta» no se define por el dispositivo eyaculatorio: la actividad es determinada por la alimentación. ¿Masa elástica - la «gelatina», también «goma»- por efecto de la lluvia que «no soportan», contrastando con el «viento» crepuscular/otoñal? La dinámica ahora es formulada por léxico como «expandirse», «digerir», «insinuarse» y «asimilar». Propagación y alimentación omnívora, ya que incluye especies de los tres reinos: «insectos», «hojas», «arena».

¿Término de la actividad de la «gisandra muerta»? El párrafo que vengo citando incluye además un nuevo lance de dados, un nuevo turn of the screw: «De año en año, las especies ya son raras o desaparecen: el jardín presiente la vegetación uniforme y degenerada (uniendo los tres reinos en la gisandra futura)»(63). El trabajo alimenticio de la «gisandra muerta» es degenerativo: esteriliza la reproducción de las especies que, así, se vuelven «raras»y «desaparecen». Es de suponer que, «aliment[ándo]se de si misma» los ciclos vuelvan a repetirse aún pero reduciendo progresivamente lo que contenga de regeneración de la ciclicidad. Esto significa que la degeneración es finita, y la interpelación de ese límite se hace mediante el sintagma «gisandra futura». Después de las «gisandras» y de la «gisandra muerta», adviene la «gisandra futura» que es del orden de un presentimiento. La antevisión de la «gisandra futura» presenta dos atributos o predicados nuevos: por un lado, la «vegetación uniforme y degenerada»y, por otro, aquel (diabólico) «uniendo los tres reinos» que traduce, de modo imperfecto, el sintagma «acopulando os três reinos» en portugués. ¿Cómo leer estos últimos sintagmas? La «gisandra futura» es, por un lado, vegetal que indiferencia otras especies vegetales (es «uniforme»). ¿Y en el caso de los «tres reinos»? ¿Nos dice el texto que en la «gisandra futura»se indiferencian, también, los «tres reinos»? No exactamente, según creo. ¿Cómo interpretar el neologismo «acopulando» — traducido imperfectamente por 'uniendo'- que amalgama «copular» $\mathrm{y}$ «acoplar»? Si colegimos las acepciones principales de «copular» tendremos 'acasalar' y 'juntar algo a otra cosa'. En el caso de «acoplar», tenemos 'ajustar algo a un lugar', 'emparejar' (ej. dos animales), 
164 Tropelías. Revista de Teoría de la Literatura y Literatura Comparada, 25 (2016)

Pedro Serra

'unir dos circuitos', 'acordar discordancia' (ej. de ideas) o 'juntar personas para actividad coordinada' ¿Acopular los «tres reinos» en la «gisandra futura» es indiferenciarlos o diferenciarlos? ¿O es la indiferencia de la diferenciación? Incognitus scriptor, el texto no decide, mantiene la cuestión en suspensión: texto en trance, pues.

\section{Bibliografía}

De Man, Paul (1996): Aesthetic Ideology. Ed. Andrzej Warminski. Minneapolis-London, University of Minnesota Press.

Mourão, Luis (1996): Um Romance de Impoder. A Paragem da História na Ficção Portuguesa Contemporânea. Braga-Coimbra, Angelus Novus.

OliveIRA, Carlos de (1978): Finisterra. Paisaje y poblamiento. Ed. Xavier Rodríguez Baixeras. Oviedo, KRK, 2010. 